\title{
Chapter 47 \\ What Do Red and Yellow Autumn Leaves Signal for Sure?
}

\begin{abstract}
Autumn leaf defensive signaling of whatever type can benefit both trees and shrubs and their herbivorous insects. At a time when the phloem sap is rich with the nutrients remobilized from senescing leaves to branch and trunk tissues, defensive, and even aposematic signaling by colorful leaves (Archetti 2000; Hamilton and Brown 2001; Archetti and Brown 2004; Lev-Yadun et al. 2004a; Lev-Yadun 2006a, 2009a; Lev-Yadun and Gould 2007; Archetti et al. 2009a) could well benefit the tree by deterring phloem sap feeders, such as aphids (e.g., Dixon 1998). Lev-Yadun and Gould (2007) proposed that autumn leaf colors may also serve as a visual warning that the leaves are going to be shed soon, reliable, honest and critical information about a significant risk of insect mortality. This is vital information for insects that need leaves that will remain on the tree for longer than several days to use as a habitat, especially for those insects that cannot climb back from the ground. Leaf fall is a well known considerable agent of insect mortality (Faeth et al. 1981), and various insects may refrain from occupying leaves that are soon to abscise (Glinwood and Pettersson 2000; Karban 2007). Thus, the signaling tree will incur less herbivory, and the insects less mortality. It is already known that certain aphid species will lay eggs on trees whose leaves have just started to change color and are yellow-orange, but they usually refrain from occupying trees with red leaves (Furuta 1986). Thus the potential for such plant-insect communication by autumn leaf coloration exists. Interestingly, Holopainen et al. (2010) found that leaves of Betula pendula that are about to be shed, emit Cis-3-hexenol, an indicator of cellular disintegration, which becomes a dominant volatile just before leaf abscission. This, combined with the yellow color, may indicate to aphids that the leaves are close to shedding.
\end{abstract}

